\title{
EVALUACIÓN DEL DAÑO POR CARBURIZACIÓN DE UN TUBO RETIRADO DE SERVICIO DE UN HORNO DE PIRÓLISIS
}

\author{
EVALUATION OF DAMAGE BY CARBURIZATION OF A TUBE REMOVED \\ FROM A PYROLYSIS FURNACE
}

\author{
Jaqueline Saavedra Rueda \\ Ing. Química, M. Sc .Ingeniería Química, Instituto Colombiano del Petróleo, \\ ECOPETROL S.A, Piedecuesta, Colombia, jaqueline. \\ saavedra@ecopetrol.com.co \\ Juan Carlos Amézquita García \\ Ing. Químico, Universidad Industrial de Santander, Colombia, \\ Juancarlosamezquita@hotmail.com \\ Laura María Díaz Burgos \\ Ing. Química, Universidad Industrial de Santander, Colombia, \\ Ladibu10@hotmail.com \\ Dionisio Laverde Cataño \\ Ing. Metalúrgico, Doctor Ingeniería Metalúrgica, \\ Universidad Industrial de Santander, Colombia, \\ dlaverder@uis.edu.co \\ Francisco Javier Bolívar Osorio \\ Ing. Metalúrgico, Doctor Ingeniería Metalúrgica, \\ Universidad de Antioquia, Colombia, \\ fbolivar@udea.edu.co \\ Francisco Javier Pérez Trujillo \\ Químico, Doctor Ingeniería Metalúrgica, \\ Universidad Complutense de Madrid, España, \\ fjperez@quim.ucm.es
}

Fecha de recepción: 15 de octubre de 2010

Fecha de aprobación: 2 de diciembre de 2010

\section{RESUMEN}

La pirólisis de hidrocarburos es un proceso que se caracteriza por llevarse a cabo durante largos períodos de tiempo a temperaturas superiores a los $843^{\circ} \mathrm{C}$ y atmósferas altamente reductoras. Dichas condiciones severas contribuyen al deterioro por 
carburización de los serpentines de los hornos en donde ocurre el proceso de rotura térmica por pirólisis, de los hidrocarburos. En este trabajo, se evaluó el estado de daño de un tramo de serpentín de la zona radiante de un horno de pirólisis de etano para la producción de etileno. El tubo fue retirado del servicio del horno de pirólisis debido a la coquización presente en su interior. Posteriores análisis de Microscopia Electrónica de Barrido, análisis metalográfico, análisis de composición química, análisis de dureza y microdureza, permitieron evaluar el estado de daño por carburización.

Palabras clave: pirólisis, carburización, microestructura, HP40, corrosión.

\section{ABSTRACT}

Hydrocarbon pyrolysis is a process characterized by carried out for long periods at temperatures above $843^{\circ} \mathrm{C}$ and environment highly reducing. These severe conditions contribute to deterioration by carburización of the coils of the furnaces which carries out the process of thermal breakage by pyrolysis of hydrocarbons. In this work it was evaluated the damage state of a section of the coil in the radiant zone of a pyrolysis furnace of ethane to ethylene production. This tube was removed from service by coking. Further analysis of Electronic Scanning Microscopy, metallographies, chemical composition analysis, hardness and microhardness, allowed to evaluate and to establish the state of damage by carburizing.

Keywords: pyrolysis, carburization, microstructure, HP40, corrosion.

\section{INTRODUCCIÓN}

La pirolisis o rotura térmica de hidrocarburos es el rompimiento de moléculas de la carga en cadenas hidrocarbonadas de menor peso molecular. A altas temperaturas, los hidrocarburos se vuelven inestables y se descomponen en hidrógeno, metano, olefinas (etileno), y aromáticos. Las reacciones de pirólisis o craqueo térmico son reacciones principalmente de deshidrogenación y rotura del enlace químico. Estas reacciones de naturaleza endotérmica se clasifican en:

Reacciones principales: se fraccionan las moléculas de la carga, formando radicales libres que favorecen reacciones en cadena hasta alcanzar el producto deseado, en este caso el etileno [1].

$$
\begin{aligned}
& \mathrm{C}_{2} \mathrm{H}_{6} \rightarrow 2 \mathrm{CH}_{3}^{*} \\
& \mathrm{CH}_{3}+\mathrm{C}_{2} \mathrm{H}_{6} \rightarrow \mathrm{CH}_{4}+\mathrm{C}_{2} \mathrm{H}_{5} \cdot \\
& \mathrm{C}_{2} \mathrm{H}_{5} \cdot \underset{\mathrm{C}_{2} \mathrm{H}_{4}+\mathrm{H}^{*}}{ }
\end{aligned}
$$


Reacciones secundarias: estas reacciones son indeseables en el proceso, por que involucran la descomposición del etileno producido y la formación de moléculas grandes como ciclo-diolefinas y aromáticos que son promotores de la formación de coque.

Las condiciones de servicio propias del proceso de pirólisis de etano para la producción de etileno, generan un ambiente agresivo para el material del serpentín, que ocasiona fenómenos perjudiciales como carburización, daño por termofluencia (creep), oxidación y carburización catastrófica (metal dusting), los cuales son el resultado de la interacción entre la atmósfera del proceso y el material de los tubos.

El ambiente en el interior de los tubos de los hornos de pirólisis, promueve el depósito de carbono en la superficie interna de los tubos, y su posterior difusión hacia el interior del material que los conforma, lo cual conduce a cambios en la microestructura y en las propiedades de la aleación.

DESCRIPCIÓN DEL HORNO DE PIRÓLISIS. El diseño típico de los hornos de pirólisis es tipo cabina, como se observa en la figura 1.

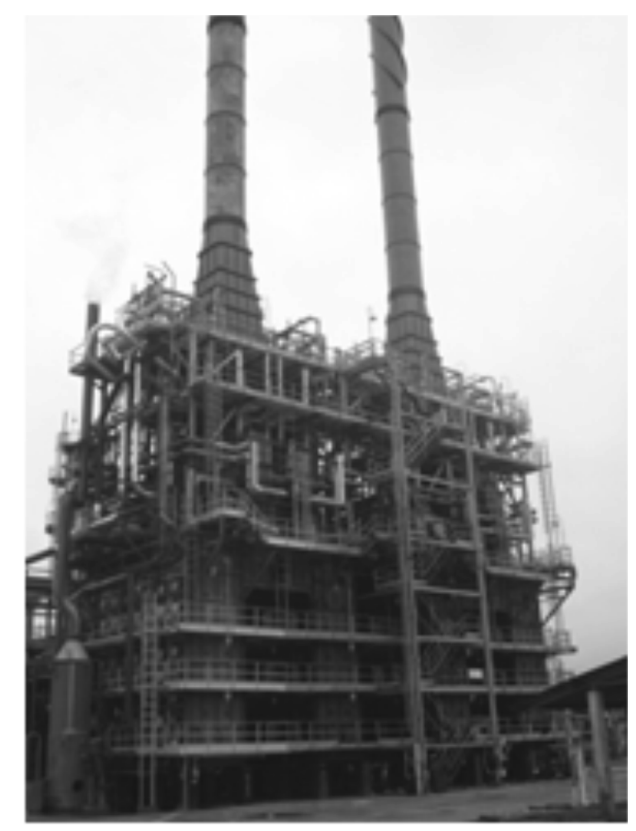

Figura 1. Horno de pirólisis de etano para producción de etileno

El horno se divide en dos zonas, una de convección y otra de radiación. En la zona de convección, se precalienta la alimentación y en la zona de radiación, ocurren las reacciones de pirólisis térmica, figura 2. 


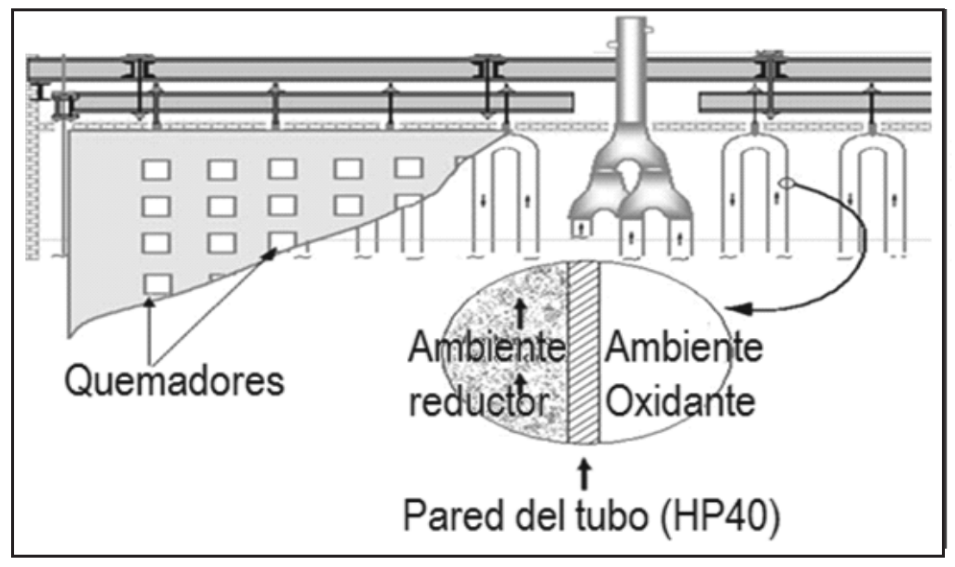

Figura 2. Zona radiante de un horno de pirólisis de etano para la producción de etileno donde ocurre el fenómeno de carburización

Debido a las altas temperaturas de esta zona, la tubería es fabricada con materiales de gran resistencia a la corrosión como el acero HP40.

La aleación HP40 tiene una microestructura formada por una matriz austenítica, compuesta básicamente por una solución aproximada de $25 \% \mathrm{Cr}, 35 \% \mathrm{Ni}, 0.4 \mathrm{C} \%$ [2], con una vida de diseño de unos 11,4 años (100000h), a una temperatura de servicio de $1173 \mathrm{~K}\left(900^{\circ} \mathrm{C}\right)$. El cromo en la aleación, mejora su resistencia a la corrosión y al daño por termofluencia.

La carburización se presenta en los tubos de los hornos de pirólisis de etano para la producción de etileno [3], los cuales están expuestos a ambientes con carbono a altas temperaturas y donde el carbono es transferido desde la atmósfera del proceso hacia el interior de la matriz metálica de la tubería.

En el mecanismo de carburización, los átomos de carbono difunden a través de la matriz metálica de un material [4], [5]. El carbono difundido puede permanecer sin reaccionar o reaccionar con los elementos constituyentes de la aleación para formar carburos $M_{23} C_{6}$ y $\mathrm{M}_{7} \mathrm{C}_{3}(\mathrm{M}: \mathrm{METAL})$, intergranulares e intragranulares, principalmente con el cromo, afectando la homogeneidad estructural del material.

La carburización es uno de los mecanismos de daño más críticos en procesos de hidrocarburos a alta temperatura [6], que afecta propiedades como el coeficiente de expansión térmica, magnetización, conductividad térmica, dureza y ductilidad, entre otras [7]. 


\section{PROCEDIMIENTO EXPERIMENTAL}

Se emplearon técnicas de análisis destructivo y no destructivo, con el fin de evaluar el estado de daño por carburización de un tubo retirado de servicio de la zona radiante de un horno de pirólisis de etano para la producción de etileno que presentó una capa de coque, figura 3. En el momento del retiro, el material llevaba 72.000 horas de trabajo.

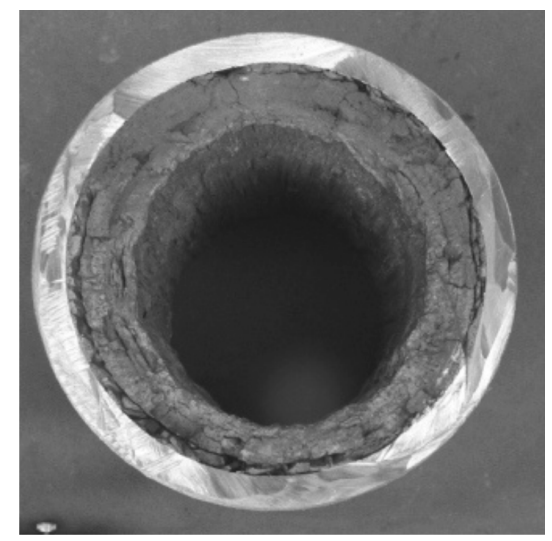

Figura 3. Tubo con coque. Material de estudio tomado de la planta petroquímica

\subsection{ENSAYOS NO DESTRUCTIVOS}

Los ensayos no destructivos que se realizaron fueron:

- Dimensionamiento: se midió el diámetro externo y se comparó con el del tubo original.

- Inspección visual: se examinó el tubo en busca de abombamientos y grietas, para determinar las zonas que pudieran presentan mayor daño.

- Magnetización: se realizó prueba cualitativa del comportamiento magnético.

\subsection{ENSAYOS DESTRUCTIVOS}

Se realizaron ensayos destructivos de composición química, dureza, microdureza y metalografía.

- Análisis de composición química del material: se hizo mediante la técnica de Espectrometría de Emisión Óptica (EEO) según norma ASTM E - 415-08 [8].

- Análisis de Dureza: se realizaron ensayos de dureza en escala Brinell - HB según norma ASTM E 10-08 [9], con una incertidumbre de la medición: $\pm 1.31 \mathrm{HB}$, en un durómetro Brinell Gnehm Horgen, aplicando 187,5Kgf. 
Para los ensayos de microdureza y metalografía, se prepararon las muestras según norma ASTM E3 [10]:

- Análisis metalográfico: realizado con ataque electrolítico de ácido oxálico al $10 \%$ a $6 \mathrm{~V}$ durante 10 segundos [11], según norma ASTM E-45.

- Análisis de Microscopia Electrónica de Barrido SEM: este análisis se realizó con un microscopio electrónico Leo1450VP, equipado con sistema de rayos x por energía dispersa OXFORD INCA [12].

- Análisis de Microdureza Vickers: estos ensayos se realizaron con un microdurómetro Shimadzu HMV 2000, aplicando 200gf, según norma ASTM E384-10e2 [13], con una incertidumbre de la medición: $\pm 6 \mathrm{HV}$

\section{RESULTADOS Y DISCUSIÓN}

\subsection{ENSAYOS NO DESTRUCTIVOS}

El espécimen analizado presentó oxidación leve en la superficie exterior (figura 4a), no presentó abombamientos, grietas o variación diametral, tabla 1. En el interior del tubo, después de retirar el coque, se observó una superficie no uniforme con desprendimiento de láminas delgadas de orientación heterogénea (figura 4b). El coque retirado del tubo presentaba tres capas; de estas, las capas en contacto con el tubo y el ambiente eran porosas, y la capa intermedia era compacta. La oxidación de la superficie externa se debe a la exposición del material a la atmosfera generada por los quemadores de la zona de radiación del horno [14].

Tabla 1. Resultado del análisis visual

\begin{tabular}{|c|c|c|c|}
\hline $\begin{array}{c}\text { Diámetro } \\
\text { externo }(\mathbf{c m})\end{array}$ & $\begin{array}{c}\text { Espesor del tubo } \\
(\mathbf{c m})\end{array}$ & $\begin{array}{c}\text { Espesor capa de } \\
\text { coque }(\mathbf{c m})\end{array}$ & $\begin{array}{c}\text { Diámetro de } \\
\text { sección libre }(\mathbf{c m})\end{array}$ \\
\hline $12,5 \pm 0,0001$ & $0,9 \pm 0,001$ & $1,8 \pm 0,0001$ & $7,1 \pm 0,001$ \\
\hline
\end{tabular}

El material evaluado mostró una respuesta magnética leve y mayor en la superficie interna que en la externa, indicando un cambio en la microestructura y en la estructura ya que la aleación tiene originalmente un comportamiento paramagnético [15, 11]. Dicho cambio se debe a la formación de carburos de cromo con el carbono que ha ingresado desde el ambiente del proceso hacia el interior del material, variando la distribución del cromo en la matriz de la aleación [15]. 


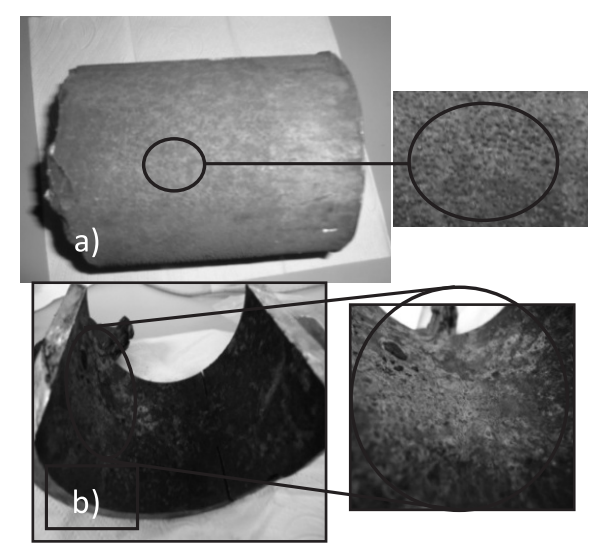

Figura 4. a) Oxidación de la superficie externa del tubo retirado de servicio

b) Superficie interna del tubo en contacto con el coque

\subsection{ENSAYOS DESTRUCTIVOS}

Con los resultados del análisis químico (tabla 2), se verificó que el material en estudio corresponde a un acero HP40, según la norma ASTM A 297 [16], [17], y se evaluó la relación cromo/carbono del material, que es más baja en el acero HP40 retirado de servicio que en el acero HP40 nuevo, debido al carbono que ha difundido hacia el interior del material, tabla 3.

Tabla 2. Composición del aceroHP40 de estudio y según norma ASTM A 297

\begin{tabular}{|l|c|c|c|c|c|c|c|}
\hline Elemento $\%$ & $\mathbf{C}$ & $\mathbf{M n}$ & $\mathbf{S}$ & $\mathbf{S i}$ & $\mathbf{N i}$ & $\mathbf{C r}$ & $\mathbf{F e}$ \\
\hline HP40 & 0.541 & 0.883 & 0.001 & 1.050 & 34.27 & 24.28 & Balance \\
\hline Grado HP 40 & $0.35-0.75$ & 2.00 Max. & 0.04 Max. & 2.50 Max. & $33.0-37.0$ & $24.0-28.0$ & Balance \\
\hline
\end{tabular}

Tabla 3. Comparación de la relación $\mathrm{Cr} / \mathrm{C}$ entre el tubo de material HP 40 retirado de servicio y el acero HP40 nuevo.

\begin{tabular}{|l|c|}
\hline \multicolumn{1}{|c|}{ Material } & Relación $\mathbf{C r} / \mathbf{C}$ \\
\hline Acero HP40 retirado del horno & 44.88 \\
\hline HP40 nuevo & 62.5 \\
\hline
\end{tabular}

El carbono que se difunde es el que está disponible en el medio, en este caso, se encuentra en forma de coque, el cual se deposita sobre la superficie interna del material gracias a la generación de carbono libre en el ambiente mediante cualquiera de las siguientes reacciones [18], [19]: 


$$
\begin{aligned}
& \mathrm{CO}+\mathrm{H}_{2}=\mathrm{C}+\mathrm{H}_{2} \mathrm{O} \\
& 2 \mathrm{CO}=\mathrm{CO}_{2}+\mathrm{C} \\
& \mathrm{CH}_{4}=\mathrm{C}+2 \mathrm{H}_{2}
\end{aligned}
$$

Donde las ecuaciones (4), (5) y (6), son las reacciones de gas de síntesis, de Boudouard y de descomposición de metano, respectivamente.

El carbono depositado termina por ingresar en el interior de la matriz del material por fenómenos de difusión en estado sólido, cuando alcanza la energía de activación necesaria para el transporte de átomos.

La termodinámica de este fenómeno de corrosión causado por gases carbonosos o hidrocarburos, es consecuencia del gradiente de concentración de carbono entre la superficie en donde se deposita el carbono y la matriz metálica de la aleación. La cinética de este proceso es lenta y depende principalmente de la temperatura [7]. En las figuras 5a y $5 \mathrm{~b}$ se muestran las microestructuras de un material HP40 nuevo [20] y la del material retirado de servicio, respectivamente.

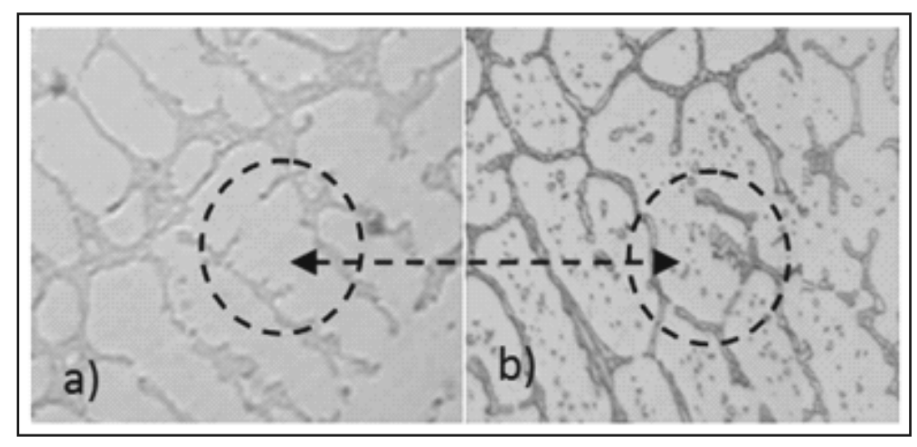

Figura 5. a) Microestructura de un material HP40 nuevo

b) Microestructura del material HP40 retirado de servicio a $100 \mathrm{X}$

Fuente: 4a) [20], 4b) Autores

La aleación HP40 tiene una microestructura formada por una matriz austenítica con precipitación de carburos eutécticos en los contornos de las dendritas como se aprecia en la figura $4^{\mathrm{a}}$; en cambio en la figura $4 \mathrm{~b}$, se observan carburos precipitados en el interior de los granos que no son propios de la aleación HP40 nueva. Esta diferencia se debe al carbono que se ha difundido hacia el interior del material durante el tiempo de servicio del tubo.

En la figura 6a, se muestra una microscopia del espécimen de estudio. En la figura $6 \mathrm{~b}$ se observa una zona de mayor aglomeración de carbono cerca de la superficie interna del tubo (aglomeración de puntos blancos), debido al carbono que se ha difundido desde el ambiente del proceso hacia el interior del material. En la figura $6 \mathrm{c}$, en los lugares donde 
hay mayor concentración de cromo (aglomeración puntos oscuros), hay carburos de este elemento por que el cromo difunde hacia los límites de grano y hacia la superficie externa del material para formar carburos $\mathrm{M}_{23} \mathrm{C}_{6}$ y $\mathrm{M}_{7} \mathrm{C}_{3}$ [21].
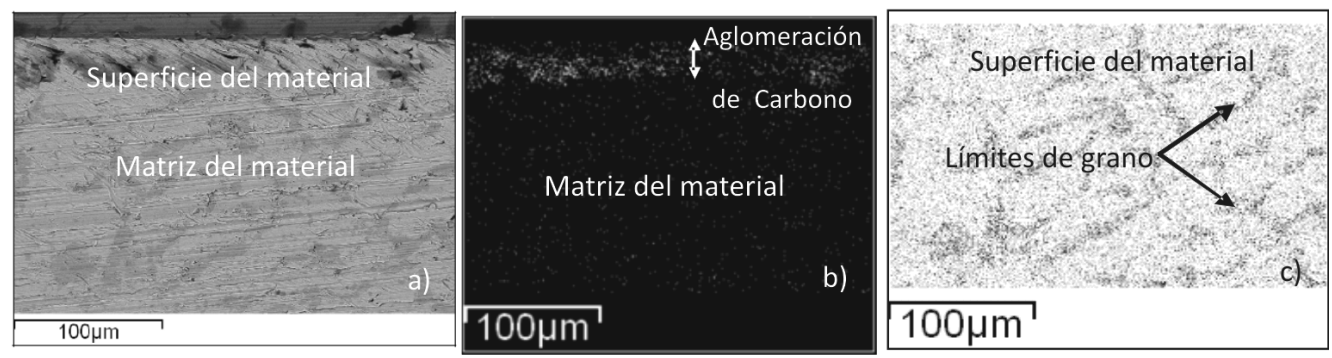

Figura 6. a) Microscopia del espécimen de estudio, b) zona de mayor aglomeración de Carbono cerca a la superficie interna del tubo, c) lugares de mayor concentración de Cromo

La dureza promedio para el acero retirado de servicio es de 188 HB (tabla 4), la cual está por encima del valor de la aleación nueva (170 HB) [22], e indica que el material ha sufrido cambios microestructurales tales como la coalescencia de carburos en los límites de grano que disminuyen la ductilidad del material.

Tabla 4. Dureza superficial del material HP40 de estudio y nuevo

\begin{tabular}{|c|c|c|c|c|}
\hline Muestra & \multicolumn{3}{|c|}{ Dureza Brinell (HB) } & Promedio \\
\hline HP40 nuevo & \multicolumn{4}{|c|}{170} \\
\hline HP40 en estudio & 188 & 188 & 189 & 188 \\
\hline
\end{tabular}

Se determinó la microdureza del material a diferentes longitudes desde la superficie interna del tubo, con el fin de observar posibles variaciones con respecto de la posición. Sin embargo, los valores de microdureza del material de estudio son uniformes con un promedio de $190 \mathrm{HMV}$, como se muestra en la tabla 5; el máximo valor presentado a las $50 \mu \mathrm{m}$ puede deberse a que los valores a esta distancia fueron tomados en un límite grano o en un carburo, los cuales tienen una dureza mayor que la matriz del material.

Tabla 5. Microdureza del material de estudio

\begin{tabular}{|c|c|c|c|c|c|c|c|c|}
\hline Posición $(\mu \mathrm{m})$ & 10 & 20 & 30 & 40 & 50 & 60 & 70 & 80 \\
\hline Dureza Vickers & 183 & 179 & 174 & 174 & 205 & 183 & 188 & 190 \\
\hline
\end{tabular}

El tubo se encuentra en buen estado general al no presentar grietas, abombamientos o deformación diametral. Sin embargo, el material mostró cambios en propiedades físicas como magnetismo, dureza y microdureza. Estos cambios se deben al carbono que ha difundido desde el ambiente carburizante del proceso hacia el interior del material, donde 
se precipita en los granos y principalmente en límites de grano, formando carburos de cromo, niobio y hierro. Este proceso genera zonas pobres en elementos como cromo y niobio, los cuales han difundido hasta los lugares en que hay precipitación de carburos. La coalescencia de carburos en los límites de grano y el empobrecimiento de las zonas adyacentes inducen esfuerzos de tensión.

El porcentaje de vida residual del material es del $80 \%$, ya que presenta carburización (precipitación de carburos intragranulaes e intergranulales), no catastrófica sin presencia de microgrietas [2]. De continuar la exposición del material en un ambiente carburizante, los esfuerzos generados por los carburos contribuirán a la formación de microgrietas, con la consecuente disminución en el porcentaje de vida residual del acero.

\section{CONCLUSIONES}

La respuesta magnética, el incremento en la dureza superficial y el cambio en la microestructura y en la estructura del material del tubo por precipitación uniforme de carburos en los bordes de dendritas y límites de grano con ausencia de grietas o abombamientos indican que el material se encuentra en un estado de daño inicial por carburización.

Usando pruebas complementarias como análisis metalográfico, dureza, microdureza y ensayos no destructivos, se pudo evaluar el estado de daño del material después de 72.000 horas de servicio para conocer su porcentaje de vida residual.

Una respuesta magnética en una acero austenítico como el HP40, indica cambios microestructurales y estructurales del material debidos a la redistribución del cromo y a la formación de nuevas fases en el interior de la aleación.

La degradación microestructural del material se debe a que el cromo de la aleación difunde hacia la superficie externa para formar óxidos y carburos, y hacia los límites de grano para formar carburos, mientras que el carbono difundido desde la atmósfera del proceso, afecta la relación cromo/carbono.

\section{REFERENCIAS BIBLIOGRÁFICAS}

[1] HM Tawancy (1993). Structure and properties of high-temperature alloys: applications of analytical electron microscopy. Dhahran (Saudi Arabia): KFUPM. Press., pp 98-99.

[2] Kaishu Guan, Hong Xu, Zhiwen Wang (2005). Quantitative study of creep cavity area of HP40 furnace tubes. En: Nuclear Engineering and Design, Vol. 235, pp. 1447-1456. 
[3] C. D. B. Meadowcroft y J. E. Oakey (1995). Guidelines for Plant Measurement of High Temperature Corrosion. En: European Federation of corrosion Publications, Vol. 14, pp. 1-9

[4] Damage Mechanisms Affecting Fixed Equipment in the Refining Industry, APIRP-571, Recommended practice 571, December 2003. En: American Petroleum Institute, First edition, pp. 3-270.

[5] ASTM G79-83 (Reapproved 1996). Standard Practice for Evaluation of Metals Exposed to Carburization Environments1.

[6] T. Maeda, F. X Terwijn (2005). Carburization resistance of high-CR, high-Ni Weld overlayed furnace tubes for ethylene pyrolysis. En: Ethylene Producers Conference, Vol 14. Session 47.

[7] H. M. Tawancy (2009). Degradation of mechanical strength of pyrolysis furnace tubesby high-temperature carburization in a petrochemical plant. En: Engineering Failure Analysis, Vol. 16, Issue 7 pp. 2171-2178.

[8] ASTM E - 415-08 Standard Test Method for Atomic Emission Vacuum Spectrometric Analysis of Carbon and Low-Alloy Steel1.

[9] ASTM E 10-08 "Standard Test Method for Brinell Hardness of Metallic Materials".

[10] ASTM E-3 de 2001Rv 07 "Standard Practice for Preparation of Metallographic Specimens".

[11] ASM American Society for Materials (1985). En: Metal Handbook, Properties and selection: Stainless steel. Vol 3.

[12] ECOPETROL, (2010). Instituto Colombiano del Petróleo ICP, Laboratorio de microscopia electrónica, Instituto Colombiano del Petróleo. Informe 10000048 ID0146 T67 10 103-ECP, Piedecuesta.

[13] ASTM E384-10e2. Standard Test Method for knoop and Vickers hardness of materials.

[14] B. Terry, J. Wright, D. Hall (1989). A model for prediction of carburization in steels for ethylene production furnaces. En: Corrosion Science, Vol 29, Issue 6, pp. 717-734.

[15] I.C. Silva, J.M.A. Rebello, A.C. Bruno, P.J. Jacques, B. Nystend and J. Dillee (2008). Structural and magnetic characterization of a carburized cast austenitic steel. En: Science Direct, Scripta Materialia, Vol 59. Issue 9, pp. 1010-1013. 
[16] ASTM A-297 de 1997 (R-2003). Standard Specification for Steel castings, IronChromium and Iron-Chromium-Nickel, Heat Resistant, for General Application.

[17] ECOPETROL S.A., (2010). Instituto Colombiano del Petróleo ICP, Laboratorio de Ingeniería de materiales. Ensayos de laboratorio a dos tuberías metálicas, Piedecuesta

[18] R. Kirchheiner, J. L. Jimenez Soler (2001). Correlation and Control of Oxidation, Carburization, and Metal Dusting in High Performance Alloys for the Petrochemical Industry. En: Corrosion 2001 NACE International, paper 01374.

[19] J. Aguilar, U. Ortiz, J. Cavazos, R. Viramontes, (1995). Carburización de aleaciones HP40+Nb y AISI 304 bajo mezclas H2, H2O, CO, CO2 Y CH4. En: México. Evento: XVII Simposio Nacional de Siderurgia.

[20] D. Alessio, (2009). Microestructura y propiedades a altas temperaturas en el acero hp 40 modificado. En: SAM-CONAMET, pp, 1091-1097.

[21] P. Jacquet, D.R. Rousse, G. Bernard, M. Lambertin (2002). A novel technique to monitor carburizing processes. En: Materials Chemistry and Physics, Vol 77, Issue 2, pp. 542-551.

[22] R. Joseph Davis. ASM specialty handbook HEAT RESISTANT MATERIALS, ASM international, pp 203. 